\title{
Identifying Gamma\#Ray Burst Remnants in Nearby Galaxies
}

\section{Citation}

Perna, Rosalba, John Raymond, and Abraham Loeb. 2000. “Identifying Gamma\#Ray Burst Remnants in Nearby Galaxies." The Astrophysical Journal 533 (2): 658-69. https:// doi.org/10.1086/308682.

\section{Permanent link}

http://nrs.harvard.edu/urn-3:HUL.InstRepos:41393264

\section{Terms of Use}

This article was downloaded from Harvard University's DASH repository, and is made available under the terms and conditions applicable to Other Posted Material, as set forth at http:// nrs.harvard.edu/urn-3:HUL.InstRepos:dash.current.terms-of-use\#LAA

\section{Share Your Story}

The Harvard community has made this article openly available.

Please share how this access benefits you. Submit a story.

Accessibility 


\title{
IDENTIFYING GAMMA-RAY BURST REMNANTS IN NEARBY GALAXIES
}

\author{
Rosalba Perna, John Raymond, and Abraham Loeb \\ Harvard-Smithsonian Center for Astrophysics, 60 Garden Street, Cambridge, MA 02138 \\ Received 1999 April 13; accepted 1999 November 30
}

\begin{abstract}
We study the spectral signatures arising from cooling and recombination of an interstellar medium whose equilibrium state has been altered over $\sim 100$ pc by the radiation of a gamma-ray burst (GRB) and its afterglow. We identify signatures in the line diagnostics that are indicative of a photoionized GRB remnant which is $\lesssim 5 \times 10^{4}$ yr old. We estimate that at least a few such remnants should be detectable in the Virgo Cluster of galaxies. If the gamma-ray emission from GRBs is beamed to a fraction $f_{b}$ of their sky, then the expected number of Virgo remnants is larger by a factor of $f_{b}^{-1}$. Virgo remnants can be resolved with arcsecond imaging and are likely to be center-filled using narrowband filters of high-ionization lines (such as [O III] 25007 or $\mathrm{He}$ II 24686 ) and limb-brightened for lowionization lines (such as [S II] 26717 ). The nonrelativistic blast wave might be visible separately, since it does not reach the outer edge of these young photoionized remnants. The remnants should show evidence for ionization cones if the prompt or afterglow UV emission from GRBs is beamed.
\end{abstract}

Subject headings: galaxies: clusters: individual (Virgo) — gamma rays: bursts - ISM: general

\section{INTRODUCTION}

Simple synchrotron models for the afterglow emission of cosmological gamma-ray burst (GRB) sources imply an ambient gas density, $\sim 1 \mathrm{~cm}^{-3}$, which is characteristic of the interstellar medium of galaxies (e.g., Waxman 1997a 1997b; Wijers \& Galama 1999). Indeed, direct imaging of the neighborhood of well-localized GRBs revealed faint host galaxies at cosmological distances in many cases (Bloom et al. 1999 and references therein). The detection of spectral signatures that can be associated with the GRB environment is of great interest both for distance measurements and for learning about the environment itself in which GRBs occur. A knowledge of the GRBs birthplace can help to constrain the validity of a given model for their formation. At the same time, it is also of fundamental interest to know how GRBs themselves affect their environments.

The most popular models for GRB formation currently involve either the collapse of a single massive star (the socalled "hypernova scenario"; Woosley 1993; Paczyński 1998; MacFadyen \& Woosley 1999) or the coalescence of two compact objects such as two neutron stars or a neutron star and a black hole (Eichler et al. 1989; Narayan et al. 1992; Ruffert \& Janka 1999). These models could be constrained by knowing the GRB environment. Massive stars have very short lives, thus they will explode in star-forming regions, which are typically characterized by very dense environments. ${ }^{1}$ On the other hand, most merging neutron stars would be very old and would have typically traveled far from their birthplace. The scenario of compact merger progenitors could thus be suggested by medium to lowdensity environments.

We have previously shown (Perna \& Loeb 1998) that the $\mathrm{X}$-ray and UV components of the afterglow radiation create an ionized bubble of radius $\sim 100 \mathrm{pc} n_{1}^{-1 / 3}$ in the surrounding galaxy, where $n_{1}$ is the ambient density in units of 1 $\mathrm{cm}^{-3}$. On a short timescale, as long as the afterglow radi-

\footnotetext{
${ }^{1}$ Note, however, that stellar winds from a massive star could displace the ISM and create a hot, low-density bubble surrounding the star before it explodes.
}

ation is still effective to ionize, the gradual ionization of the medium can produce time-dependent absorption (Perna \& Loeb 1998; Meszaros \& Rees 1998) and emission lines (Ghisellini et al. 1998; Böttcher et al. 1998).

In this paper we compute the emission spectrum, which results as the ionized gas slowly cools and recombines. Cooling times are typically very long, $t_{\text {cool }} \sim 10^{5}\left(T / 10^{5} \mathrm{~K}\right) /$ $\left(n_{e} / 1 \mathrm{~cm}^{-3}\right) \mathrm{yr}$, at a temperature $T$ and an electron density $n_{e}$. If GRBs occur in galaxies, then their rate is estimated to be $\sim\left(10^{6}-10^{7} f_{b} \text { yr) }\right)^{-1}$ per galaxy (Wijers et al. 1998), where $f_{b} \leq 1$ is the unknown beaming factor (covering fraction) of the gamma-ray emission. This implies that in every galaxy there is a nonnegligible probability of finding an ionized GRB remnant at any given time. The identification of these remnants in nearby galaxies will allow a much closer study of the sites where GRBs occurred and will provide an estimate for the energy output and occurrence frequency of the events (Loeb \& Perna 1998).

The hydrodynamic impact of a GRB blast wave on its environment lasts longer than the radiation ionization effect. It takes tens of millions of years for the GRB blast wave to slow down to a velocity of $\sim 10 \mathrm{~km} \mathrm{~s}^{-1}$, at which point it may be erased by interstellar turbulence. Hence, old GRB remnants should consist of a large-size ( kiloparsec), expanding, cold $\mathrm{H}$ I shell, similar to the $\mathrm{H}$ I supershells that were identified for two decades in nearby galaxies (Loeb \& Perna 1998; Efremov, Elmegreen, \& Hodge 1998 and references therein). However, it appears difficult to distinguish the old hydrodynamic remnants produced by GRBs from those produced by the accumulated effect of more conventional energy sources, such as multiple supernovae (McCray \& Kafatos 1987), stellar winds from OB associations (Abbott et al. 1981), radiation pressure (Elmegreen \& Chiang 1982), or impact from high-velocity clouds (Tenorio-Tagle 1981). ${ }^{2}$ In some cases, a class of these possibilities is disfavored. For example, recent deep-CCD imaging of the $\mathrm{H}$ I holes in the Holmberg II galaxy (Rhode et al. 1999) did not reveal the anticipated optical emission from a normal stellar population in several of these holes, in

\footnotetext{
${ }^{2}$ This scenario is favoured in cases where only one hemisphere of the shell is present.
} 

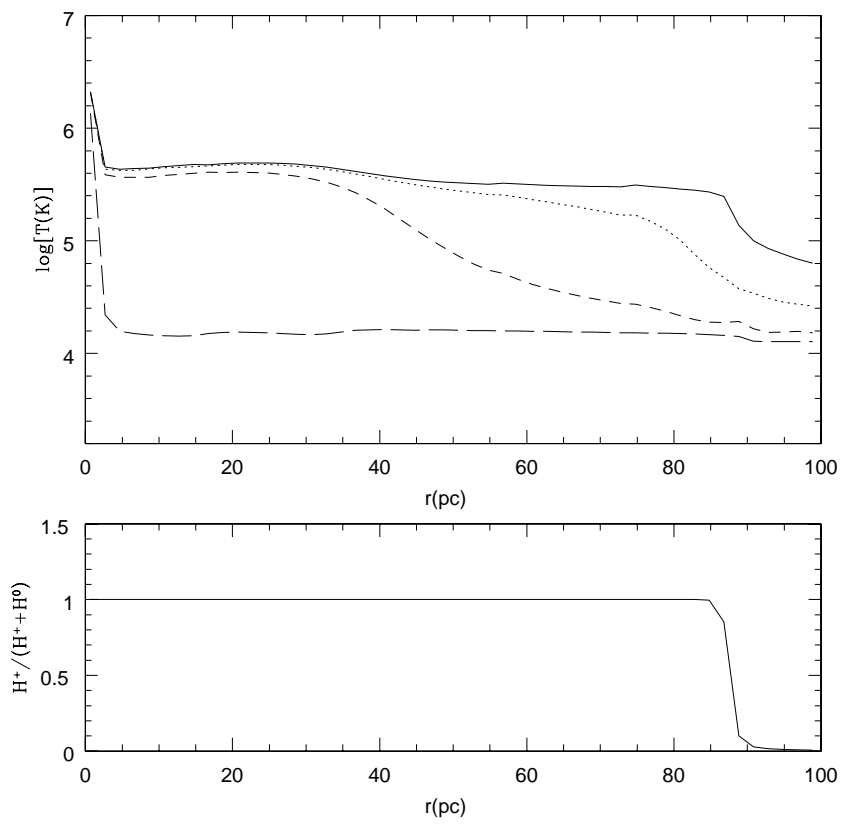
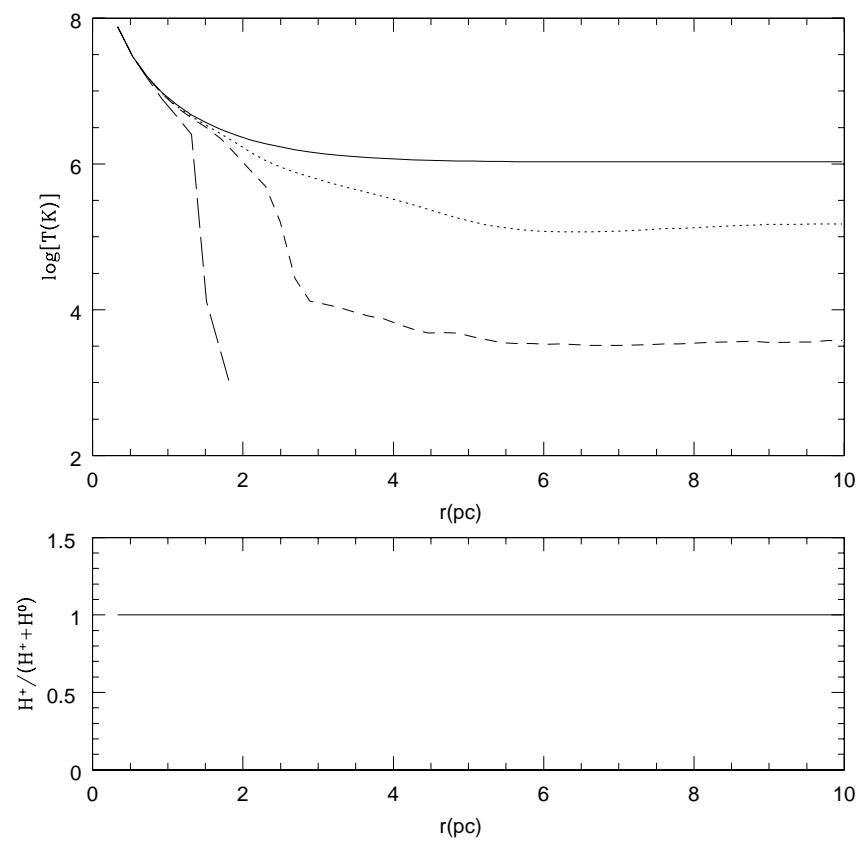

Fig. 1. - Left: Temperature profile of the remnant of a GRB of energy $10^{52} \mathrm{ergs}$ in a medium of density $1 \mathrm{~cm}^{-3}$. Here the times are $t_{\mathrm{obs}}=3 \times 10^{2} \mathrm{yr}(\mathrm{solid}$ line), $t_{\mathrm{obs}}=3 \times 10^{3} \mathrm{yr}$ (dotted line), $t_{\mathrm{obs}}=10^{4} \mathrm{yr}$ (dashed line), and $t_{\mathrm{obs}}=10^{5} \mathrm{yr}\left(\right.$ long-dashed line). Right: Ionized hydrogen fraction $\mathrm{H}^{+} / \mathrm{H}^{0}$ at the times immediately following the passage of the afterglow radiation through the shells.

conflict with the multiple supernova or stellar wind interpretations. Nevertheless, even in this case the old age of the hydrodynamic remnants does not allow for a unique identification of GRBs as their energy sources. On the other hand, since the energy release in GRB remnants is impulsive, it should be easier to distinguish them from conventional sources by identifying their unique spectral signatures at a sufficiently early time when they are young and radiant. As we show later, the emission from young GRB remnants with ages of $\lesssim 10^{4} \mathrm{yr}$ is affected mostly by the radiative ionization effect of the early GRB afterglow on its surrounding interstellar medium, since it takes much more than $10^{4} \mathrm{yr}$ for the nonrelativistic blast wave to traverse the photoionized region. The impulsive energy release of hardionizing radiation is unique to GRB sources and could distinguish young GRB remnants from the remnants of multiple supernovae.

The goal of this paper is to identify the spectral signatures that are peculiar to GRB remnants and that can be distinguished from those due to the remnants of other explosive events, such as supernovae. In $\S 2$ we present the computational scheme adopted for this problem. In $\S 3$ we show our numerical results and analyze the particular spectral signatures of young GRB remnants. In $\S 4$ we consider the expected effect of variations in the input parameters used in our calculations. Finally, in $\S 5$ we summarize our main conclusions.

\section{MODEL ASSUMPTIONS AND COMPUTATIONAL SCHEME}

We consider a GRB source which turns on at time $t=0$ and illuminates a stationary ambient medium of uniform density $n$, with a time-dependent luminosity per unit frequency, $L_{v}(t)$. The release in the surrounding medium of a large amount of ionizing radiation is a distinctive feature of GRBs and their afterglows, as opposed to supernova explosions, where any impulsive electromagnetic release would not escape promptly, but would be degraded by adiabatic expansion of the envelope before it could leak out. In our work, we limit our analysis to the effects of the afterglow photoionizing radiation on the medium. The blast wave lags behind the ionizing front and, until the time it reaches larger radii from which most of the absorption and reemission comes, is not expected to greatly affect the ionization state of the medium and the resulting luminosity. We will discuss this point in greater detail in $\S 3$.

Afterglows are most naturally explained by models in which the bursts are produced by relativistically expanding fireballs (Paczyński \& Rhoads 1993; Meszaros \& Rees 1997; Vietri 1997a, 1997b; Waxman 1997a, 1997b; Wijers, Rees, \& Meszaros 1997; ; Sari 1997). On encountering an external medium, the relativistic shell which emitted the initial GRB decelerates and converts its bulk kinetic energy to synchrotron radiation, giving rise to the afterglow. The combined radio and optical data imply that the fireball energy is $\sim 10^{51}-10^{52}$ ergs. In the simplest unbeamed synchrotron model (e.g., Waxman 1997a, 1997b), the time and frequency dependence of the afterglow luminosity is given by

$$
L_{v}(t)=L_{v_{m}}\left[\frac{v}{v_{m}(t)}\right]^{-\alpha},
$$

where,

$$
v_{m}(t)=1.7 \times 10^{16}\left(\frac{\xi_{e}}{0.2}\right)^{2}\left(\frac{\xi_{B}}{0.1}\right)^{1 / 2} E_{52}^{1 / 2} t_{\mathrm{hr}}^{-3 / 2} \mathrm{~Hz} .
$$

Here $\xi_{B}$ and $\xi_{e}$ are the fractions of the equipartition energy in magnetic field and accelerated electrons, $E=10^{52} E_{52}$ ergs is the fireball energy $t_{\mathrm{hr}} \equiv(t / \mathrm{hr})$, and

$$
L_{v_{m}}=8.65 \times 10^{29} \sqrt{n_{1}}\left(\frac{\xi_{\mathrm{B}}}{0.1}\right) E_{52} \operatorname{ergs~s}^{-1} \mathrm{~Hz}^{-1},
$$

where $n_{1}$ is the ambient proton density in units of $1 \mathrm{~cm}^{-3}$. The spectral index $\alpha$ is chosen to have the values $\alpha_{1}=-\frac{1}{3}$ 

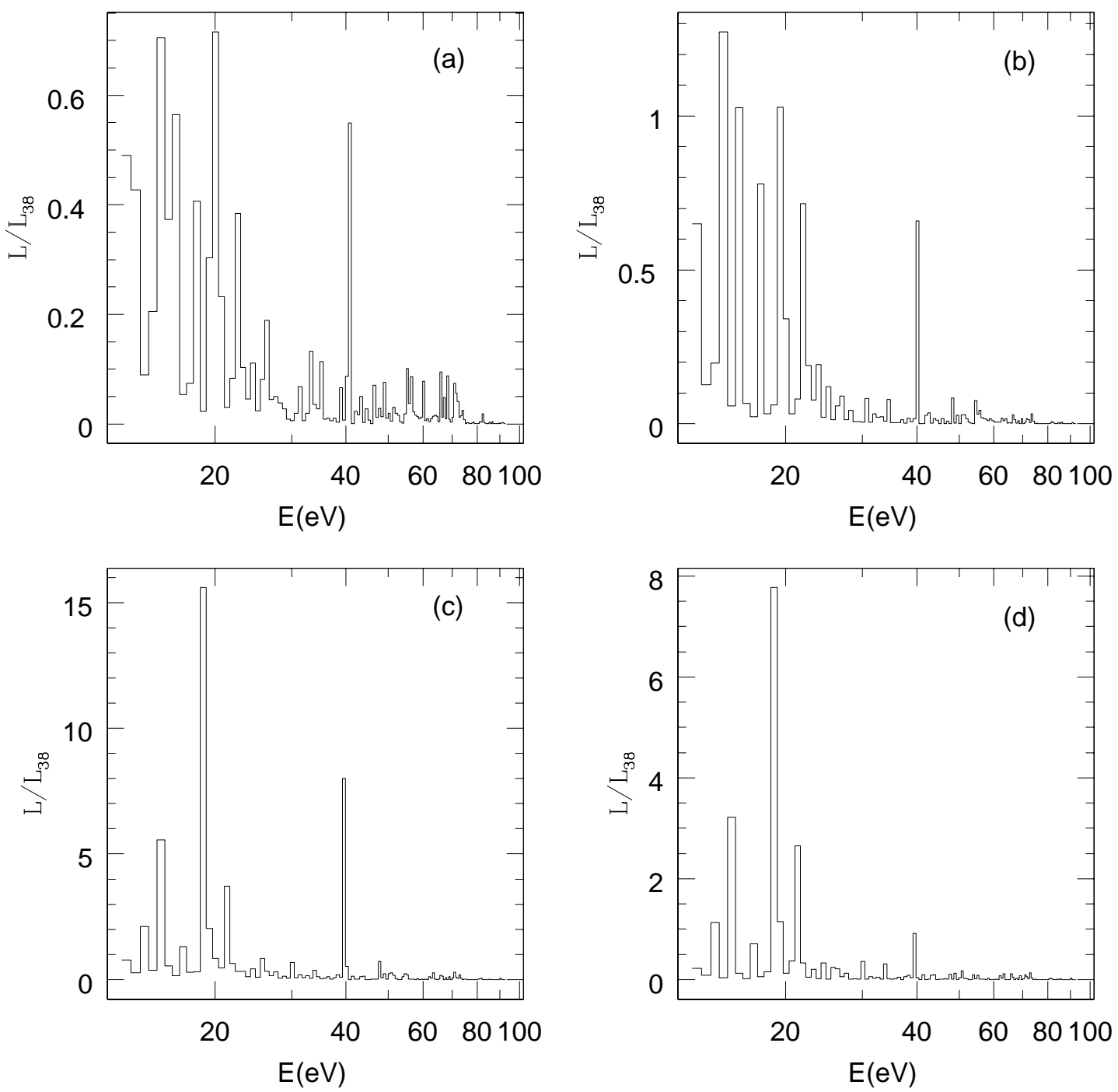

Fig. 2. - Emission spectrum above $13 \mathrm{eV}$ of the remnant of a GRB of energy $10^{52} \mathrm{ergs}$ in a medium of density $1 \mathrm{~cm}^{-3}$. Here the times are $(a) t_{\mathrm{obs}}=3 \times 10^{2}$ $\mathrm{yr},(b) t_{\mathrm{obs}}=3 \times 10^{3} \mathrm{yr},(c) t_{\mathrm{obs}}=10^{4} \mathrm{yr}$, and $(d) t_{\mathrm{obs}}=10^{5} \mathrm{yr} . L_{38}$ is the luminosity in each photon-energy bin in units of $10^{38} \mathrm{ergs} \mathrm{s}^{-1}$.

for $v \leq v_{m}$ and $\alpha_{2}=0.7$ for $v>v_{m}$, so as to match the temporal decay slope observed for GRB 970228 (Fruchter et al. 1998) and GRB 970508 (Galama et al. 1998).

We consider a uniform medium that is initially neutral and in thermodynamic equilibrium with a temperature $T \sim 10^{4} \mathrm{~K}^{3}$ and include all of the most important astrophysical elements, that is, $\mathrm{H}, \mathrm{He}, \mathrm{C}, \mathrm{N}, \mathrm{O}, \mathrm{Ne}, \mathrm{Mg}, \mathrm{Si}, \mathrm{S}, \mathrm{Ar}$, $\mathrm{Ca}, \mathrm{Fe}$, and Ni. Their abundances are taken from Anders \& Grevesse (1989). We consider a region surrounding the GRB site of size $R$ and medium density $n$, and we split it up into a radial grid with steps $\Delta r$. This is chosen so that the energy balance (between the energy lost by the afterglow and that absorbed by the gas) is maintained. In propagating from a point at position $r$ to another point at position $r+\Delta r$, the afterglow flux is reduced according to

$$
F_{v}(r+\Delta r, t+\Delta t)=F_{v}(r, t) \exp \left[-\Delta \tau_{v}(r, t)\right] \frac{r^{2}}{(r+\Delta r)^{2}},
$$

${ }^{3}$ As long as the energy absorbed by the gas is much larger than its initial thermal energy, the final temperature reached by the gas is essentially independent of the value chosen for the initial temperature. where $F_{v}$ is in units of ergs $\mathrm{cm}^{-2} \mathrm{~s}^{-1} \mathrm{~Hz}^{-1}$. We denote the local number densities of the ions of the various elements by $n_{j}^{a}(r, t)$, where the superscript $a$ characterizes the element and the subscript $j$ characterizes the ionization state. The optical depth due to photoabsorption within the distance $\Delta r$ is then given by

$$
\Delta \tau_{v}(r, t)=\Delta r \sum_{a, j} n_{j}^{a}(r, t) \sigma_{j}^{a}(v) .
$$

The photoionization cross sections are taken from Reilman $\&$ Manson (1979). The abundances of the ions of the elements are determined by solving the system of equations

$$
\begin{aligned}
\frac{d n_{j}^{a}(r, t)}{d t}= & q_{j-2} n_{j-2}^{a}+q_{j-1} n_{j-1}^{a}+c_{j-1} n_{j-1}^{a} n_{e} \\
& -\left(q_{j}+c_{j} n_{e}+\alpha_{j} n_{e}\right) n_{j}^{a}+\alpha_{j+1} n_{j+1}^{a} n_{e} .
\end{aligned}
$$

The $q_{j}$ and $c_{j}$ are the photoionization and collisional ionization coefficients of ion $j$, respectively, while $\alpha_{j}$ is the recombination coefficient. Note that $q_{j-2}$ refers to inner shell 

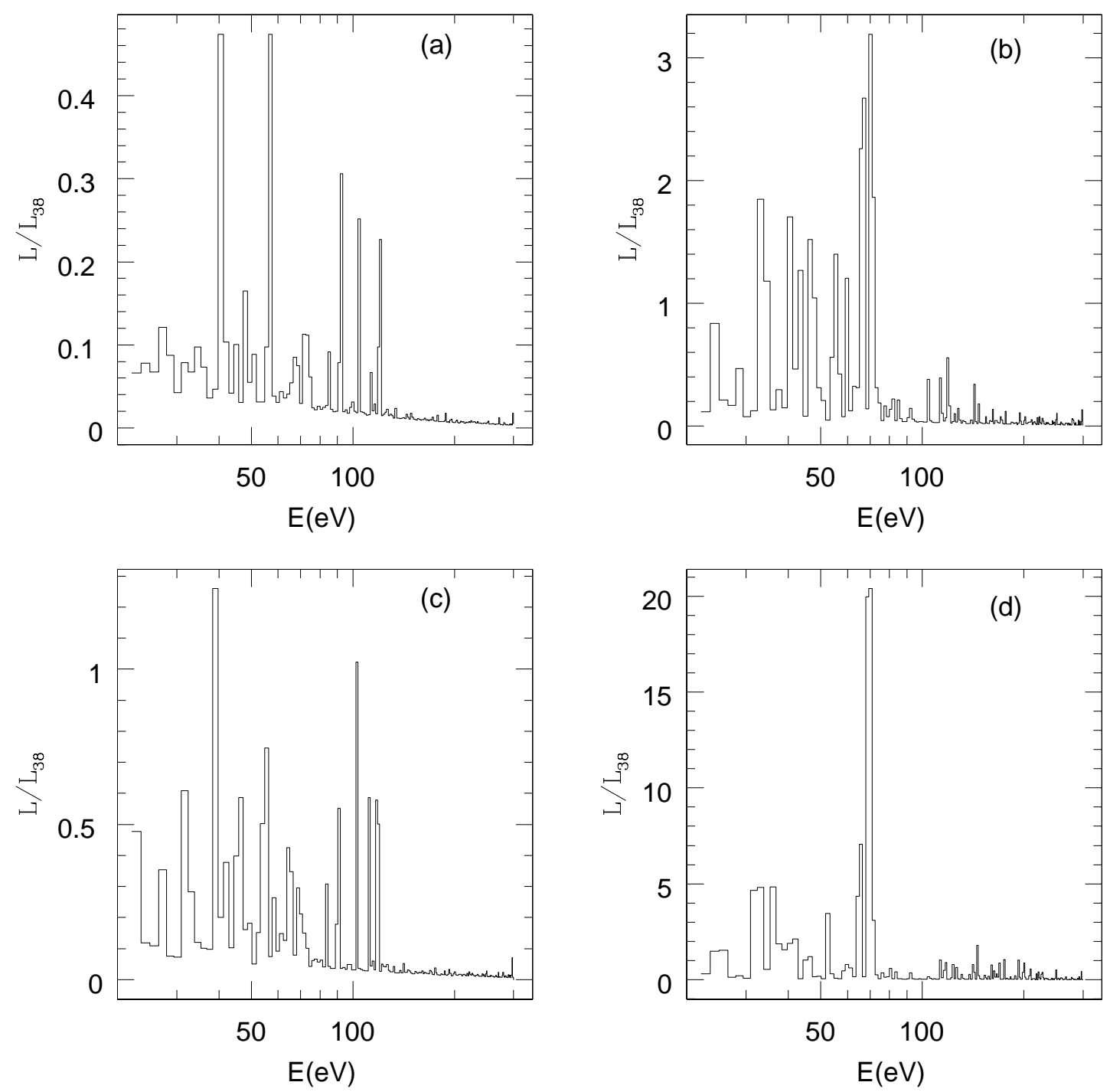

FIG. 2.-Continued

photoionization followed by Auger ionization. The collisional ionization rates are calculated according to Younger (1981). We compute the terms due to photoionization by integrating $F_{v} \sigma_{v}$ numerically. The recombination rates are given by the sum of the radiative and dielectronic recombination rates. The radiative recombination process is the inverse of photoionization, so the rates to the ground states are computed from the photoionization cross section with the help of the detailed balance relation. Hydrogenic rates are used for radiative recombination to excited levels. The dielectronic recombination rates are taken from Burgess (1965) with modifications to take more recent calculations into account. Most important is the reduction due to autoionization to excited states (Jacobs et al. 1977), with an appropriate treatment of the weakening of this effect at higher $Z$ (Smith et al. 1985). Since we are dealing with a nonequilibrium situation, the ionization fractions are calculated within the program. The emissivity of the medium, $E_{v}(r, t)$, is calculated by using the atomic rates packages of the shock model code described by Raymond (1979), and modified with updated atomic rates, as in Cox \& Raymond (1985). This code computes the spectrum of radiation emitted by a hot, optically thin plasma. The basic processes that produce the continuum radiation are bremsstrahlung, recombination, and two-photon continuum. Permitted-line radiation and the most important forbidden lines are also included, as well as the recombination-line radiation from $\mathrm{H}-$ and $\mathrm{He}$-like ions. Photoionization heating and radiative cooling are calculated within the same code and used to update the temperature of the plasma as a function of position and time. Compton heating and cooling of the electrons by the radiation is also taken into account, as well as the secondary effect of the radiation emitted by the gas on the gas itself. This effect is especially important during the late phase of cooling.

We start the simulation $(t=0)$ at a position $R_{\min } \ll R$ and let the afterglow flux propagate and evolve according to equation (4), while calculating at each position $r_{i} \leq c t$ of the grid the abundances of all the ions of each element, the temperature of the plasma, and the local emissivities $E_{v}\left(t, r_{i}\right)$. Let $t_{\mathrm{obs}}$ be the observer time, such that the radiation detected at $t_{\mathrm{obs}}=0$ corresponds to that emitted at $t=0$ in the source frame. Then a photon emitted at position $r$ at an angle $\theta$ with the line of sight will be detected by the observer at a time $t_{\mathrm{obs}}$ if it is emitted in the source frame at a time $t=t_{\mathrm{obs}}+r \cos \theta / c$. The total emitted radiation that 

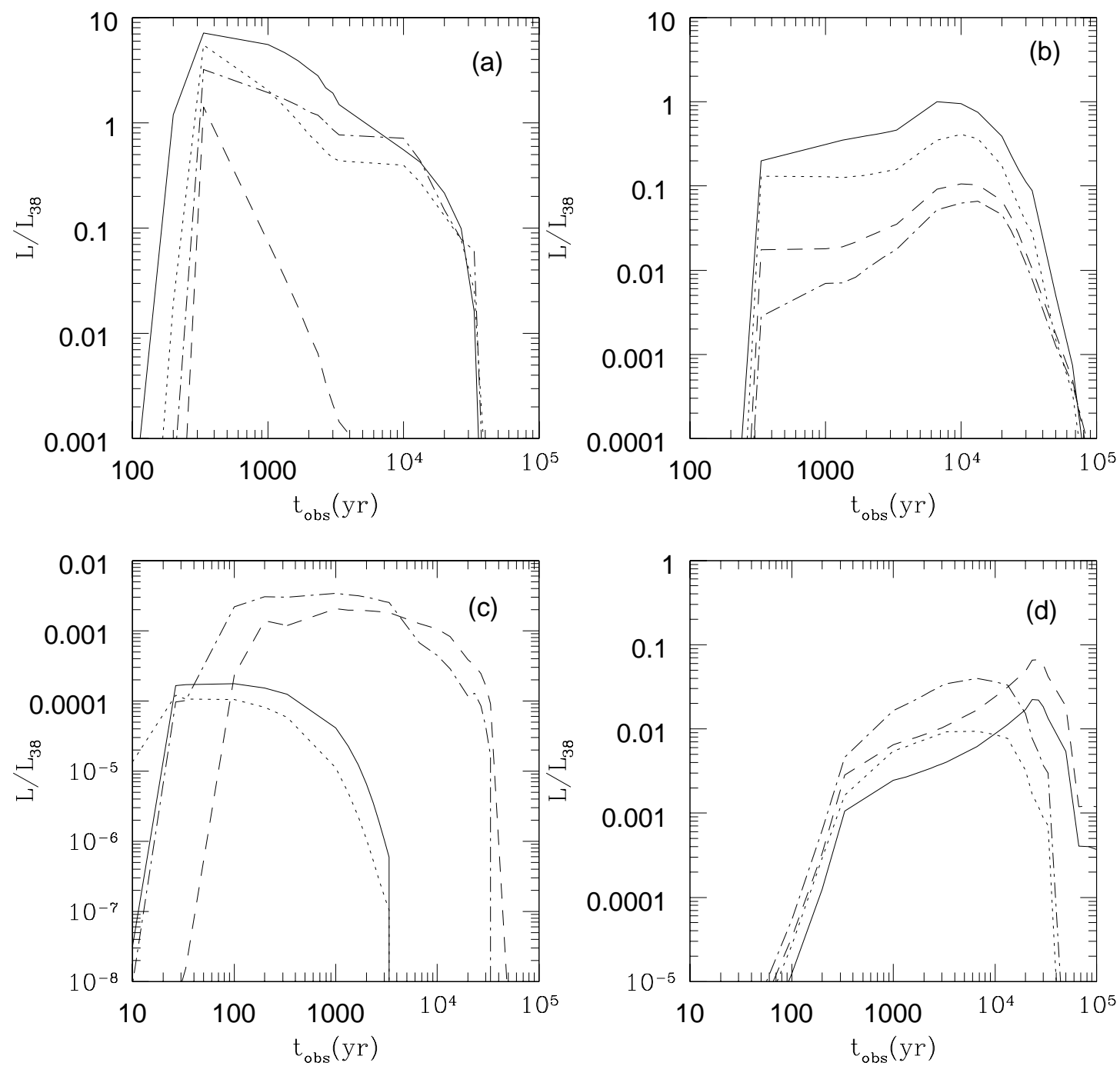

FIG. 3.-Left: Time variation of the luminosity of some of the strongest emission lines for the remnant of a GRB of energy $10^{52}$ ergs in a medium of density $1 \mathrm{~cm}^{-3}$. The lines are the following: (a) He II at $\lambda=1640 \AA \AA$ (dashed line), $\mathrm{C}$ III at $\lambda=977 \AA$ (dot-dashed line), $\mathrm{C}$ IV at $\lambda=1549 \AA$ (dotted line), and O VI at $\lambda=1034 \AA$ (solid line); (b) [O III $]$ at $\lambda=4959+5007 \AA$ (solid line), [O II $]$ at $\lambda=3729 \AA$ (dotted line), [S II $]$ at $\lambda=6717 \AA$ (dot-dashed line), and [N II $]$ at $\lambda=6548+6584 \AA$ (dashed line); (c), O VII at $\lambda=21.60 \AA$ (dashed line), $\mathrm{O}$ VIII at $\lambda=18.97 \AA$ (dot-dashed line), Fe XxV at $\lambda=1.859 \AA$ (solid line), and Fe XxVI at $\lambda=1.780 \AA$ (dotted line); (d), $\mathrm{H} \alpha$ (dashed line), $\mathrm{H}_{c} \alpha$ (dot-dashed line), $\mathrm{H} \beta$ (solid line), and $\mathrm{H}_{c} \beta$ (dotted line). Right: Time variation of the luminosity of some of the strongest emission lines for the remnant of a GRB of energy $10^{52}$ ergs in a molecular cloud of density $10^{2} \mathrm{~cm}^{-3}$ and radius $10 \mathrm{pc}$. The lines are the same as those at left.

reaches the observer at time $t_{\text {obs }}$ is given by

$$
\begin{aligned}
E_{v}^{\mathrm{tot}}\left(t_{\mathrm{obs}}\right)= & 2 \pi \int_{0}^{R_{\max }} d r r^{2} \int_{-1}^{1} d \cos \theta \\
& \times E_{v}\left(r, t_{\mathrm{obs}}+\frac{r \cos \theta}{c}\right) \\
= & 2 \pi c \int_{0}^{R_{\max }} d r r \int_{t_{\mathrm{obs}}-(r / c)}^{t_{\mathrm{obs}}+(r / c)} d t E_{v}(r, t) .
\end{aligned}
$$

\section{SPECTRAL SIGNATURES OF GRB REMNANTS}

The top panels of Figure 1 (left) and Figure 1 (right) depict the temperature profile of a GRB remnant at several times. In Figure 1 (left) we consider the situation where a GRB of energy $E=10^{52}$ ergs occurs in a typical interstellar medium, for which we assume the density $n=1 \mathrm{~cm}^{-3}$. The change in slope in the temperature profile near $3 \mathrm{pc}$ occurs when Compton heating becomes dominant over the heating due to photoionization. The drop around $85 \mathrm{pc}$, on the other hand, occurs when most of the energy carried by the UV component of the afterglow radiation (which is on the order of a few percent of the total input energy) has been absorbed.

Figure 1 (right) shows the case of a burst of the same energy $E=10^{52}$ ergs occurring in a dense cloud of density $n=10^{2} \mathrm{~cm}^{-3}$ and size $R=10 \mathrm{pc}$. As the afterglow flux is proportional to $\sqrt{n}$ (see eq. [3]), the gas is heated to a higher temperature close to the source than in the lower density case. However, it cools much faster than a lower density gas heated to the same temperature, because $t_{\text {cool }} \propto$ $n^{-1}$. In the simulation, a cutoff is set at $T=10^{3} \mathrm{~K}$, because below that temperature there is no more optical emission, and molecular gas starts to form. In Figure 1, the bottom panels show the ionized hydrogen fraction $\mathrm{H}^{+} / \mathrm{H}^{0}$ at the times immediately following the passage of the afterglow radiation through the various shells. 

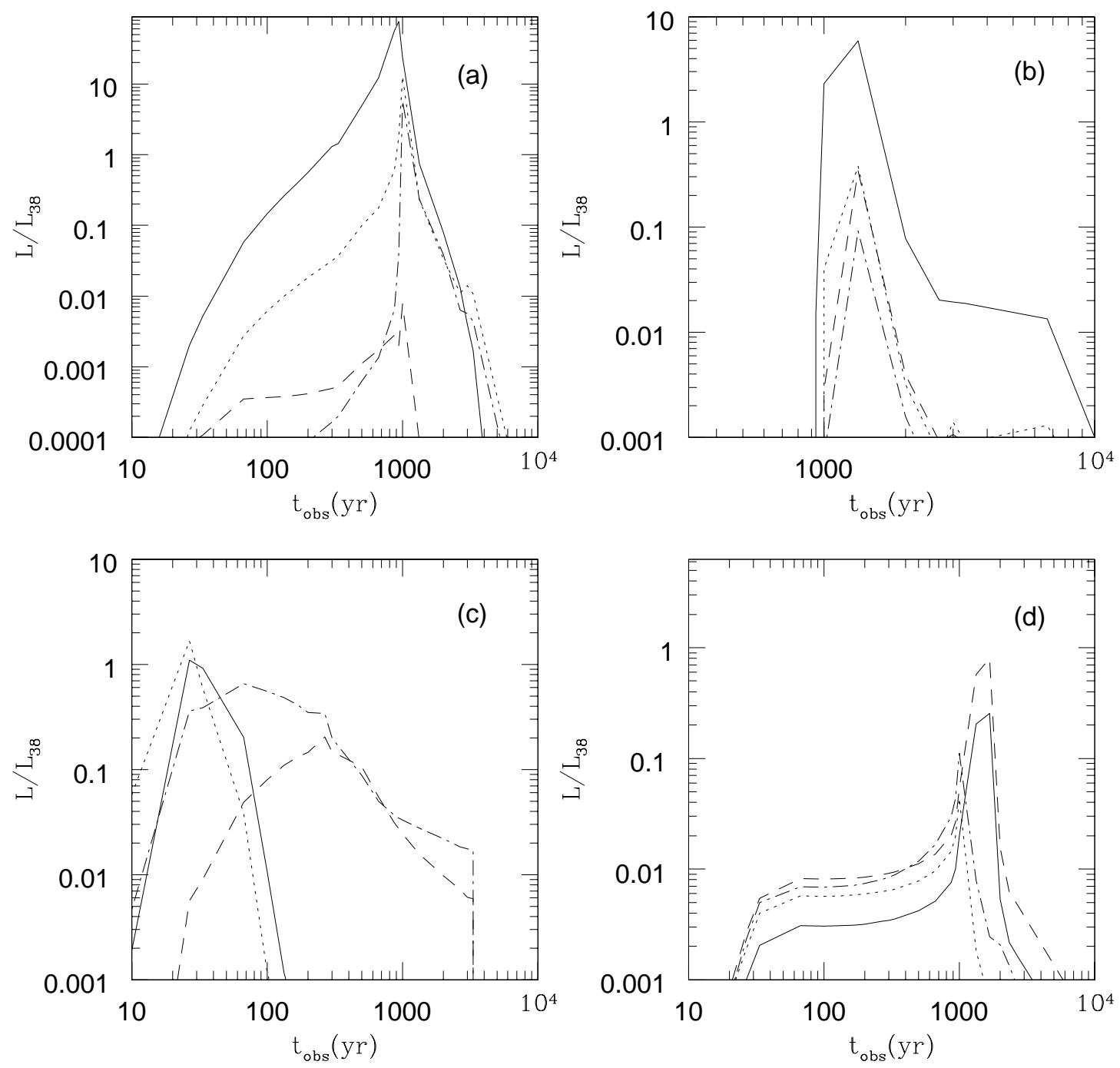

FIG. 3.-Continued

Figure 2 panels show the emission spectrum above $13 \mathrm{eV}$ at several times during cooling for the same set of parameters used in Figure 1. This ionizing flux is important for the luminosities and intensity ratios of the optical lines after the gas cools to around $10^{4} \mathrm{~K}$. The time behavior of some of the most important lines in the observable regions of the spectrum is shown separately in Figure 3 (left) and Figure 3 (right), again with the same set of parameters as in Figures 1 and 2. Notice how the emission from the remnant is very weak in the first tens of years, and rapidly rises when $t_{\mathrm{obs}} \gtrsim$ $300 \mathrm{yr}$ (particularly noticeable in panels $a$ and $b$ of Fig. 3 [left]). This is a result of the fact that the emission to the observer starts to come from the entire volume of the remnant only when $t_{\mathrm{obs}}$ becomes comparable to the light crossing time $R / c$.

Figures 2 and 3 show that the energy from a remnant in a typical interstellar medium is mostly reemitted in the optical, UV and soft X-ray band. This is to be contrasted with the emission from a young supernova remnant, where the gas, heated by the shock to temperatures $\gtrsim 10^{7} \mathrm{~K}$, produces a strong emission in harder regions of the X-ray band. Moreover, in the case of a GRB remnant, the X-ray emission comes mostly from the inner radii, whereas the UV and optical lines come from the bulk of the volume (and this is why they are several orders of magnitude brighter than the $\mathrm{X}$-ray lines). To make this more explicit, in Figure 4 we plot the abundances of some of the ions of oxygen as a function of radius at different times. It can be seen how most of the volume (except for the inner radii) contributes to the $\mathrm{O}$ III emission, whereas the limb is brightened in $\mathrm{O}$ II. In the high-density case, the lifetime of the emission lines is shorter (see Fig. 3 [right]) due to the rapid cooling of the dense gas. Here for the high density case, we considered a cloud of size $10 \mathrm{pc}$ (corresponding to a column density of $3 \times 10^{21}$ $\mathrm{cm}^{-2}$ ). Much higher column densities are not typically inferred in GRBs. In any event, for a burst that occurs in a denser region, leaving a larger fraction of its energy in the surrounding medium, luminosities up to about 2 orders of magnitude higher than the ones shown could be observed in its remnant.

Figure 5 shows the ratios between some strong optical lines as a function of time. Some important diagnostic plots commonly used to distinguish among various excitation mechanisms (Baldwin, Phillips, \& Terlevich 1981; Baum, Heckman, \& van Brugel 1992; Dopita \& Sutherland 1995) are shown in Figure 6.

The emission-line ratios exhibited by the nebulae reflect the mechanism by which the gas is ionized and the chemical 

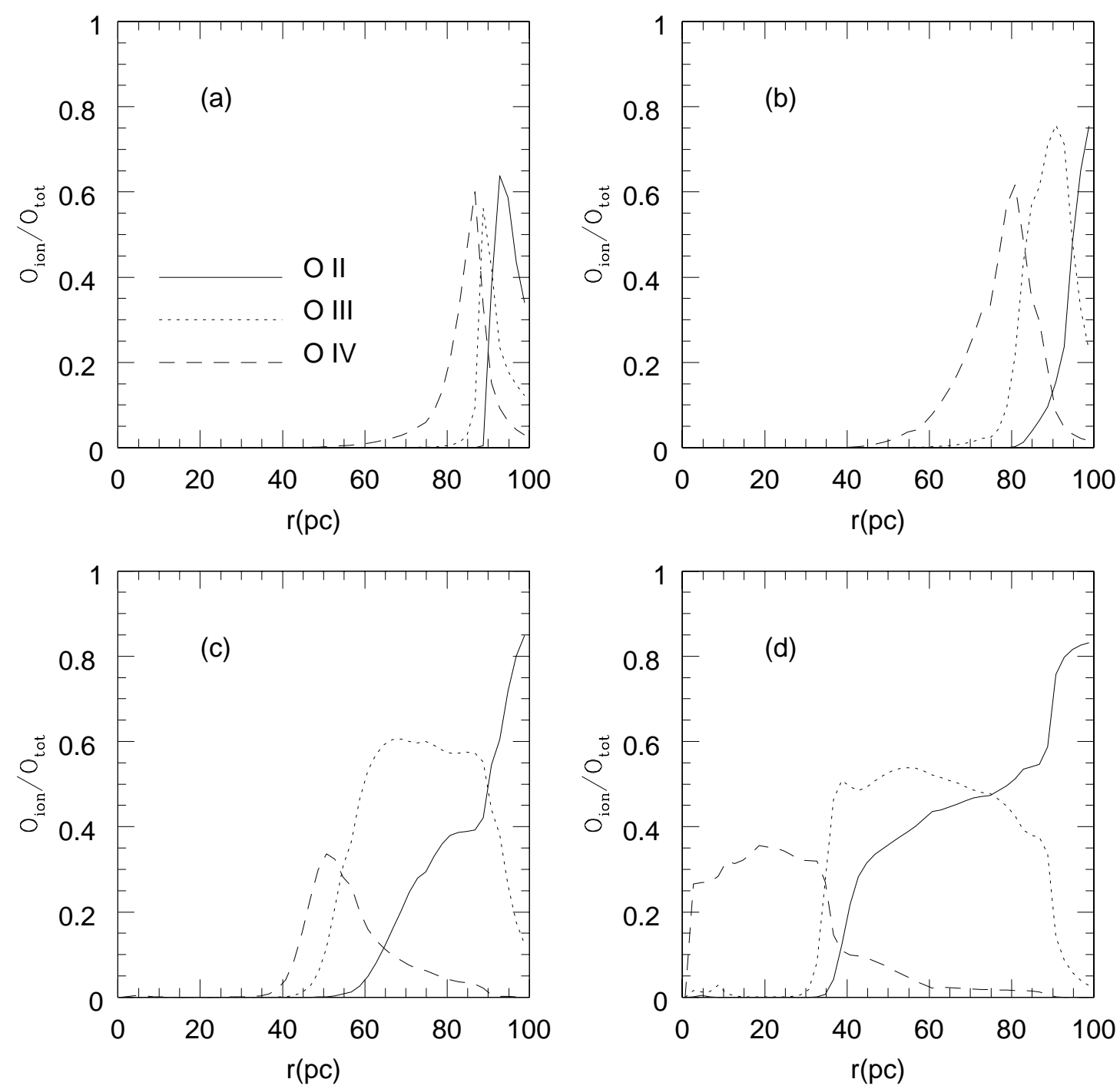

FIG. 4.-Fractional abundances of some ions of oxygen for the remnant of a GRB of energy $10^{52}$ ergs in a medium of density $1 \mathrm{~cm}^{-3}$. The times are $(a)$ $t_{\mathrm{obs}}=3 \times 10^{2} \mathrm{yr},(b) t_{\mathrm{obs}}=2 \times 10^{3} \mathrm{yr},(c) t_{\mathrm{obs}}=10^{4} \mathrm{yr}$, and $(d) t_{\mathrm{obs}}=3 \times 10^{4} \mathrm{yr}$.

abundances and physical conditions in the line-emitting gas. If the gas is purely photoionized, the ionization state of the gas is determined primarily by the ionization parameter, defined by $U=Q(H) / 4 \pi r^{2} n_{e} c$, where $Q(H)$ is the number of ionizing photons per second emitted by the source, and to a lesser extent is affected by the shape of the ionizing flux. A remarkable feature of our diagnostic plots is the generally high value of the ratio between the [O III] $\lambda 5007$ line and $\mathrm{H} \beta$. Numerical simulations (Shull \& McKee 1979) show that such high ratios (i.e., $[\mathrm{O} \mathrm{III}] \lambda 5007 / \mathrm{H} \beta \geq 5$ ) cannot be produced in shocks, but are produced by photoionization models in which the ionization parameter is relatively high (i.e., $\sim$ for $[\mathrm{O} \mathrm{III}] / \mathrm{H} \beta \simeq 10$; this ratio increases with ionization parameter). In our case, the ionization parameter is $\gg 1$ close to the source, and is higher than $\sim 10^{-3}$ for most of the ionized gas. At early times, because of time-delay effects, the bulk of the emission comes from the region close to the source with very high values of the ionization parameter, and this leads to correspondingly high values of [O $\mathrm{III}]$ $\lambda 5007 / \mathrm{H} \beta$ not typically found in regions excited by other mechanisms. Note, however, that high values of [O III] $\lambda 5007 / \mathrm{H} \beta$ are occasionally observed in supernova remnant shocks, but only during a brief period of incomplete cooling (e.g., Raymond et al. 1988) or in oxygen-rich supernova remnants (e.g., Morse et al. 1996).

The ratio between [O III] $\lambda 5007$ and [O III] $\lambda 4363$ is a diagnostic of the temperature of the emitting plasma. Its increase with time is a signature of the fact that the gas is cooling. The temperature indicated is generally far higher than is observed in steady state photoionized plasmas such as $\mathrm{H}$ II regions. It is even higher than is common in supernova remnants (Raymond et al. 1988) for much of the GRB cooling time.

Another commonly used line ratio diagnostic for nebulae is the [S II] $\lambda \lambda 6717,6727 / \mathrm{H} \alpha$ ratio, which is small in $\mathrm{H} \mathrm{II}$ regions and planetary nebulae, and $\gtrsim 0.4$ in most shocks. A GRB remnant shows the signatures of photoionization for most of its cooling time.

$\mathrm{O}$ II is in the low-density limit. The region that produces it is the edge of the photoionized region (see Fig. 4), which is excluded from the higher density model. This is why the [O II] $\lambda \lambda 3727,3729$ is lower in this model.

Perhaps the most unusual feature of the optical emission is the high ratio of $\mathrm{He}$ II $\lambda 4686$ to $\mathrm{H} \beta$. While this ratio is 

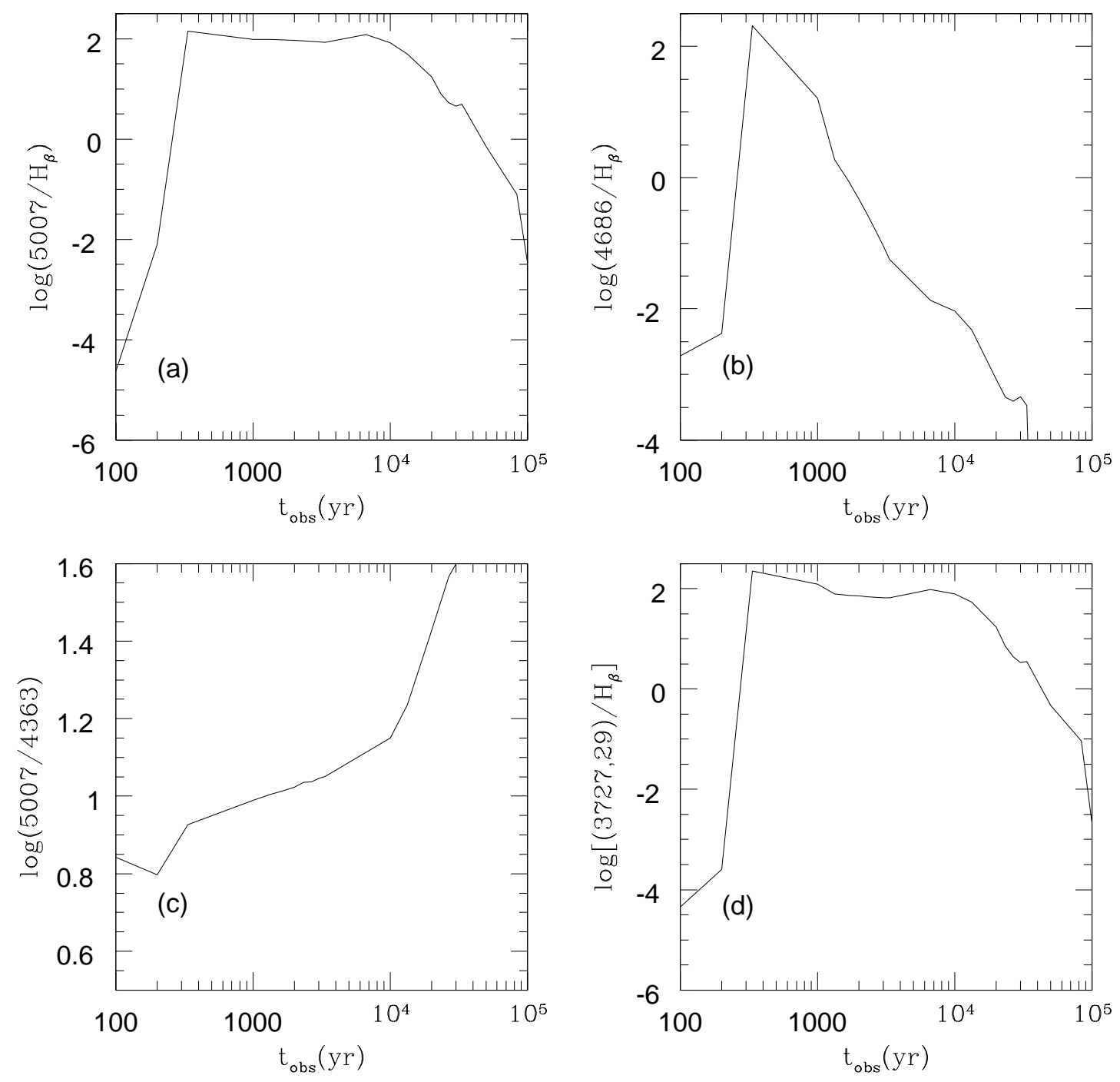

FIG. 5-Left: Time dependence of some line ratios that can be used as diagnostics for the remnant of a GRB of energy $10^{52}$ ergs in a medium of density 1 $\mathrm{cm}^{-3}$. Right: Same as Fig. 5 (left), but for the remnant of a GRB of energy $10^{52} \mathrm{ergs}$ in a molecular cloud of density $10^{2} \mathrm{~cm}^{-3}$ and radius $10 \mathrm{pc}$.

high for only a short time, the He II emission is extremely weak in $\mathrm{H}$ II regions and seldom exceeds 0.1 in supernova remnants. The strength here results from the existence of a huge volume of gas at $10^{5} \mathrm{~K}$ or more and the faintness of the Balmer lines at these temperatures.

It is true that GRB remnants spend some time in the region populated by $\mathrm{H}$ II regions as they pass from much higher ionization conditions when young to much lower ionization when old. It would be more difficult to identify a GRB remnant during this phase, but still possible if an OB association is available to power the nebula. Moreover, the UV lines and temperature-sensitive line ratios such as [O III] $\lambda 4363 / \lambda 5007$ indicate higher temperatures in GRB remnants than those found in $\mathrm{H}$ II regions.

In our simulation of the impact of a GRB on the external medium, we have considered only the effects of photoionization. As a matter of fact, a shock front lags behind, and we need to estimate how it affects the GRB signatures that we discussed. Needless to say, the photoionization model is only valid until the blast wave produced by the GRB event reaches the photoionized material. As long as the shocked gas is very hot, however, it will have little effect on the optical spectrum. The shock compresses the gas, thereby increasing its emissivity, but it also heats the gas. This tends to increase the energy emitted, but to decrease the number of photons produced. The blast wave will strongly affect the optical spectrum when: (1) it has swept up a substantial fraction of the photoionized gas or (2) when the blast wave becomes radiative, producing strong ionizing radiation and strong optical emission from the cooling gas. The former case occurs when the blast wave reaches $\frac{2}{3}$ the radius of the photoionized region (thus reducing the volume of the optically emitting volume by $30 \%$ ). The latter occurs when the shock slows to about $300 \mathrm{~km} \mathrm{~s}^{-1}$, depending upon the explosion energy only as the $1 / 11$ power (Cox 1972).

For an explosion with an energy $E_{52} \times 10^{52}$ ergs in a uniform medium of density $n_{1} \mathrm{~cm}^{-3}$, the late phase of the blast-wave evolution is described by the Sedov (1959) solution: $R \approx(19 \mathrm{pc})\left(E_{52} / n_{1}\right)^{1 / 5} t_{4}^{2 / 5}$, where $t_{4}$ is the time from the explosion in units of $10^{4} \mathrm{yr}$. The corresponding velocity of the wave is $v \approx\left(750 \mathrm{~km} \mathrm{~s}^{-1}\right)\left(E_{52} / n_{1}\right)^{1 / 5} t_{4}^{-3 / 5}$. For an explosion with $10^{52}$ ergs in a medium of density $n_{1}$, the blast wave will reach a distance of about $50 \mathrm{pc}$ after $t \approx 10^{6} \mathrm{yr}$, while the shock reaches a velocity of $300 \mathrm{~km} \mathrm{~s}^{-1}$ after $t \approx 4.6 \times 10^{4} \mathrm{yr}$. At that time, the shock has traveled a distance of about $35 \mathrm{pc}$. Let us consider the effect of the 

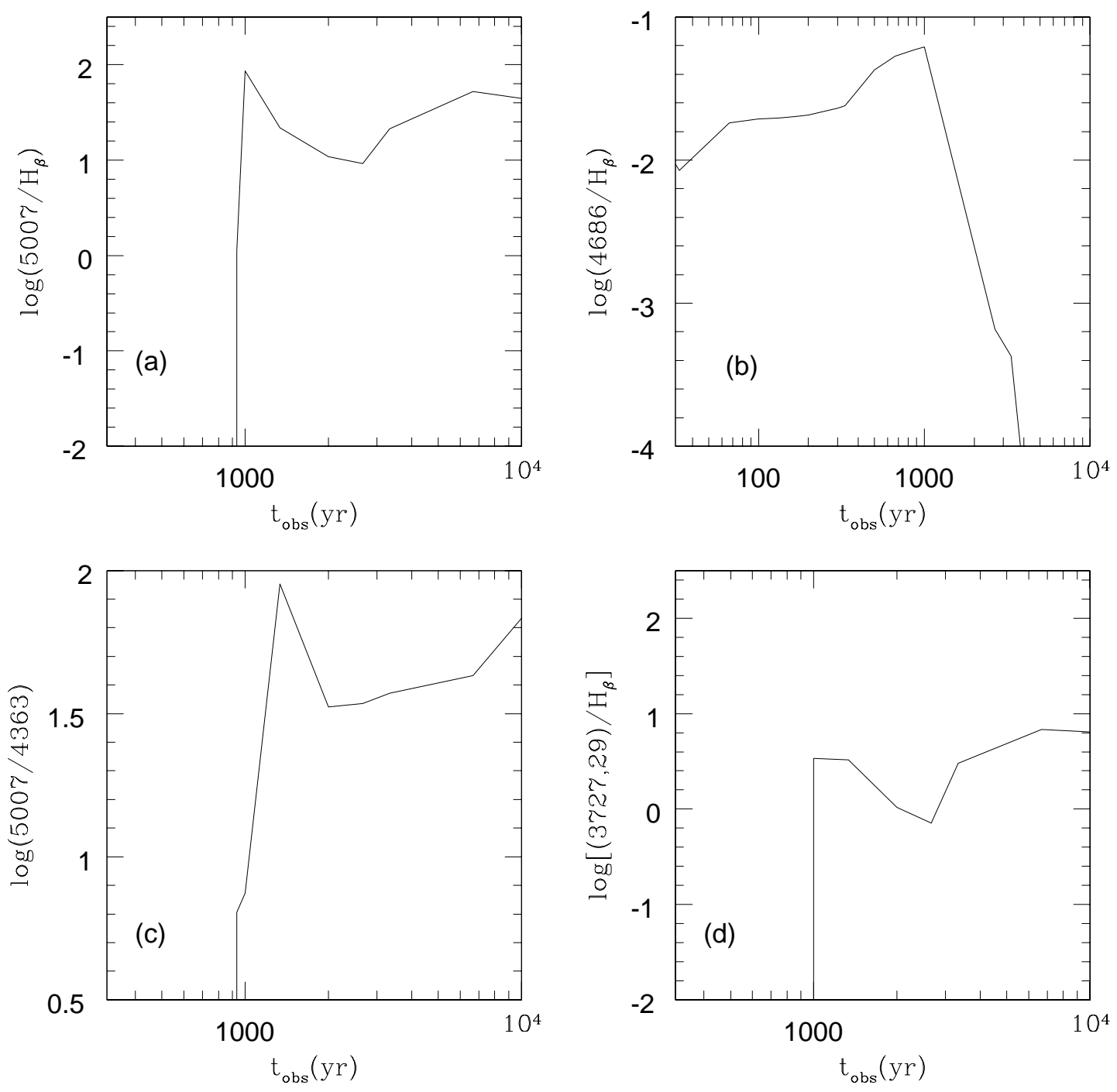

FIG. 5.-Continued

emission from the shock on a particularly important line, such as [O III] 25007 . From the simulations of Hartigan, Raymond, \& Hartmann (1987), we see that the flux in the 5007 line from a shock at a velocity of $300 \mathrm{~km} \mathrm{~s}^{-1}$ in a medium of density $1 \mathrm{~cm}^{-3}$ is $\sim 10^{-4} \mathrm{ergs} \mathrm{cm}^{-2} \mathrm{~s}^{-1}$. At a distance of $100 \mathrm{pc}$, this flux is $\sim 10^{-5} \mathrm{ergs} \mathrm{cm}^{-2} \mathrm{~s}^{-1}$ and it has to be compared with the flux from the same line due to the photoionized gas. This is on the order of $10^{38} /$ $\left(4 \pi r^{2}\right) \sim 10^{-4} \mathrm{ergs} \mathrm{cm}^{-2} \mathrm{~s}^{-1}$. Thus, the contribution of the shock to the emission when condition (2) is satisfied is only a few percent. The same analysis for the higher density case shows, instead, that after a time $\sim 10^{4} \mathrm{yr}$ the optical spectrum becomes dominated by the emission from the shock.

\section{DISCUSSION}

The model that we assumed for our GRB has a typical energy of $10^{52}$ ergs, which is released isotropically, and the afterglow is produced in the standard fireball model. However, this might not always be the real scenario. A prompt optical-UV flash was detected for GRB 990123 (Akerlof et al. 1999). If such a flash (coincident with the GRB and lasting for less than a minute) is generic in GRBs and carries as much energy as the gamma-ray emission (i.e., much more than the optical-UV afterglow emission), then
GRBs might ionize a larger region than we previously considered. The photoionization signatures would be even stronger, and the luminosities higher. If, on the other hand, the optical-UV afterglow is beamed (and thus its energy lower than commonly estimated), then GRBs have a weaker effect on their environment, and in this case it would be more difficult to distinguish them from other photoionized nebulae. A situation of nonsteady state caused by photoionization in a region where there is no evidence of a nearby photoionizing source is, however, more generally typical of a GRB remnant. Unless the progenitor of a GRB is a massive star, one does not generally expect to find GRB remnants in star-forming regions. On the other hand, photoionized nebulae are generally found around OB associations. Note that recently Wang (1999) has reported observations of X-ray-emitting regions in M101 which did not show any evidence for $\mathrm{OB}$ associations, and has made the hypothesis that they could be associated with GRB remnants.

Other complications could arise from a nonhomogeneous medium. If the medium has dense clumps in it, then these will absorb more flux than the surrounding region; they will be more luminous, but will cool faster. Depending on the pressure gradient at its boundary, a dense clump 

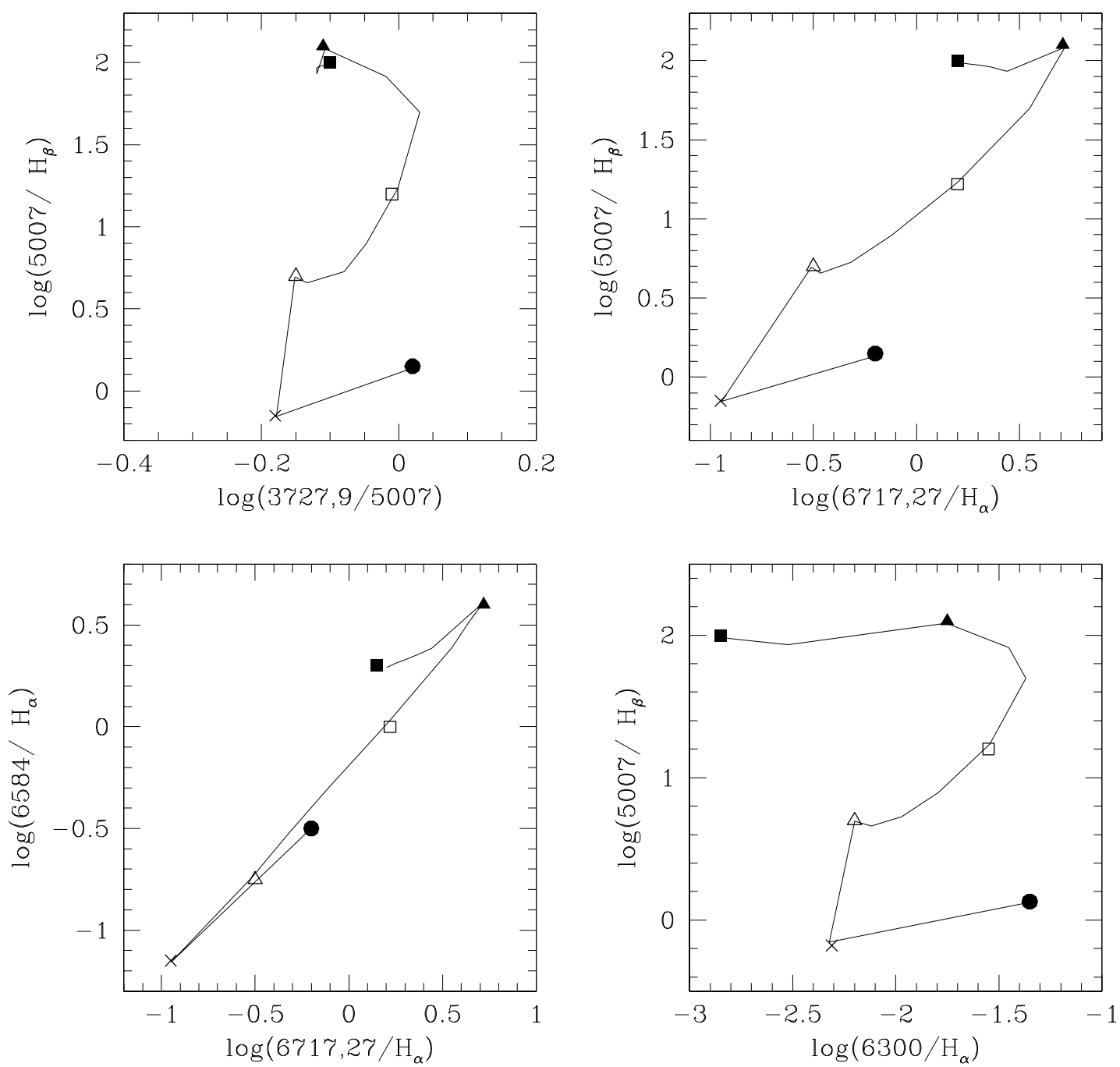

FIG. 6. - Left: Line diagnostics for the remnant of a GRB of energy $10^{52}$ ergs in a medium of density $1 \mathrm{~cm}^{-3}$.. Some of the times are indicated by a special symbol: $t_{\mathrm{obs}}=10^{3} \mathrm{yr}$ ( filled square) $; t_{\mathrm{obs}}=6 \times 10^{3} \mathrm{yr}$ (filled triangle) $t_{\mathrm{obs}}=2 \times 10^{4} \mathrm{yr}$ (empty square) $; T_{\mathrm{obs}}=4 \times 10^{4} \mathrm{yr}($ empty triangle $) ; t_{\mathrm{obs}}=5 \times 10^{4} \mathrm{yr}$ (cross); and $t_{\mathrm{obs}}=7 \times 10^{4} \mathrm{yr}$ ( filled circle). Right: Same diagnostics as in Fig. 6 (left), but for the remnant of a GRB of energy $10^{52}$ ergs in a molecular cloud of density $10^{2} \mathrm{~cm}^{-3}$ and radius $10 \mathrm{pc}$. The times indicated here by a special symbol are the following: $t_{\mathrm{obs}}=10^{3} \mathrm{yr}($ filled square $) ; t_{\mathrm{obs}}=2 \times 10^{3} \mathrm{yr}($ filled triangle $)$; and $t_{\mathrm{obs}}=7 \times 10^{3} \mathrm{yr}$ (filled circle) .

may expand (and cool adiabatically) or suffer additional compression. Small clumps are more likely to be heated to a comparable temperature with respect to the surrounding medium, and thus they will expand, due to the higher pressure caused by their higher density. On the other hand, a large dense clump will absorb a considerable amount of flux and, thus, will show a much steeper temperature gradient with respect to the lower density surrounding medium. In this case the clump might expand in one direction and be compressed in another. The time dependence of the luminosity as a function of time in these cases would show more complicated patterns. Modeling all these secondary effects is beyond our scope, especially because the real conditions of the medium are unknown and the possible early production of the afterglow and its degree of beaming are as yet a subject of debate. Also, it is still far from clear whether there is a unique scenario for all the bursts or if, instead, there can be substantial differences from one burst to another.

Our final discussion needs to address the issue of how many of these GRB remnants are detectable with current instruments. To this purpose, let us make some rough estimates of the possibility of detection of a strong line, say the [O III] $\lambda 5007$, for example. Its luminosity is $\gtrsim 10^{37} \mathrm{ergs} \mathrm{s}^{-1}$ for a time $t_{L} \approx 4 \times 10^{4} \mathrm{yr}$. The corresponding flux of photons at a distance $d_{\mathrm{Mpc}} \times \mathrm{Mpc}$ is $F_{\text {signal }}=2 \times 10^{-2} d_{\mathrm{Mpc}}^{-2}$ $\mathrm{cm}^{-2} \mathrm{~s}^{-1}$. The emission from the host galaxy around this wavelength constitutes the main source of background noise. The number of photons for a band of $20 \AA$ around the $5007 \AA$ wavelength from the inner region (out to around 5 $\mathrm{kpc}$ ) of an $\mathrm{Sb}$ galaxy, for example, is $F_{\text {noise }} \approx 5 \times 10^{-5}$ $\mathrm{cm}^{-2} \mathrm{~s}^{-1} \operatorname{arcsec}^{-2}$ (D. Fabricant, private communication 1999). For a telescope with a diameter $D=10 \mathrm{~m}$, a spectroscopic detection efficiency of $\epsilon=0.1$, and an integration time of $t_{\mathrm{int}}=10 \mathrm{hr}$, the signal-to-noise ratio $(\mathrm{S} / \mathrm{N})=$ $N_{\text {signal }} /\left(N_{\text {noise }}+N_{\text {signal }}\right)^{1 / 2}$ and obtains a value of $\sim 1.5$ $\times 10^{5} d_{\mathrm{Mpc}}^{-2}\left(1+4 \times 10^{2} d_{\mathrm{Mpc}}^{-2}\right)^{-1 / 2}$, where $N=\epsilon F\left(\pi D^{2} / 4\right) t_{\text {int }}$ is the number of photons detected if a $1^{\prime \prime}$ resolution element is assumed. An $\mathrm{S} / \mathrm{N} \geq 10$ will thus correspond to a maximum detection distance of $d_{\max } \approx 200 \mathrm{Mpc}$. This distance is an order of magnitude larger than the distance to the Virgo Cluster of galaxies $\left(d_{\mathrm{Virgo}} \approx 16 \mathrm{Mpc}\right)$ and is com- 

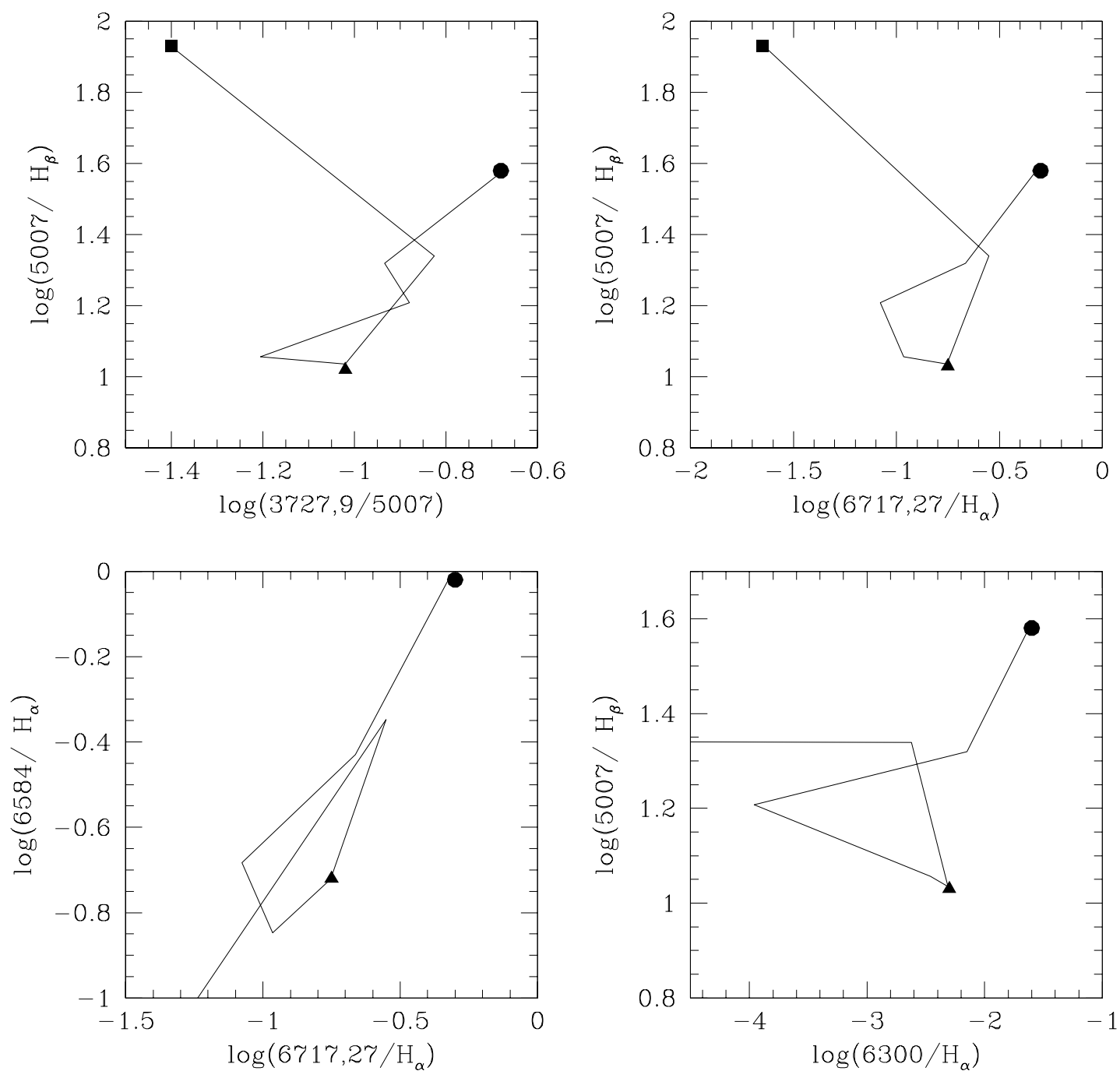

Fig. 6.-Continued

parable to the distance of Coma cluster $\left(d_{\text {Coma }} \approx 10^{2} \mathrm{Mpc}\right)$. Hence, let us estimate the number of remnants that could be detected. For a population of GRBs that follow the star formation history, Wijers et al. (1998) estimated the local rate per galaxy to be $\Gamma_{\mathrm{GRB}}=2.5 \times 10^{-8} \mathrm{yr}^{-1}$. Multiplying this number by $t_{L}$ yields a probability of about $10^{-3}$ of finding a young remnant per galaxy. The number of galaxies in the Virgo Cluster up to a magnitude $B=19$ is about 2500 and the total number of bright $\left(L_{*}\right)$ galaxies out to a distance of $200 \mathrm{Mpc}$ is $\sim 10^{5}$. Thus, a few GRB remnants could be easily detected in the Virgo Cluster, and about a hundred are detectable out to the limiting distance of $200 \mathrm{Mpc}$. We note that the above estimate is sensitive to the redshift distribution of GRBs; for example, if GRBs constitute a nonevolving population, the estimated GRB rate per galaxy is about 40 times higher (Fenimore \& Bloom 1995).

If the gamma-ray emission from GRBs is beamed to within a fraction $f_{b}$ of their sky, then the number of remnants in Virgo would increase as $\Gamma_{\mathrm{GRB}} \propto f_{b}^{-1}$, while the maximum number of remnants out to the limiting distance would decrease as $\Gamma_{\mathrm{GRB}} d_{\text {max }}^{3} \propto f_{b}^{1 / 2}$. Hence, for a relatively modest beaming factor of $f_{b} \lesssim 0.1$, there should be more remnants observable in Virgo than elsewhere. Moreover, Virgo remnants are easier to detect because they can be resolved, while distant remnants cannot be resolved and their flux could be easily dominated by contaminating light from their host galaxy. Based on these considerations, we conclude that an effective observational search should focus on identifying young GRB remnants in the Virgo Cluster.

A photoionized remnant of radius $\sim 100 \mathrm{pc}$ at a distance of $20 \mathrm{Mpc}$ occupies an angular diameter of $2^{\prime \prime}$ on the sky and, therefore, could be resolved. The strong emission lines from such a remnant can be detected with an $S / N \approx 100$ after $1 \mathrm{hr}$ of integration on the Keck telescope. Because of the temperature decrease at outer radii, we expect such a remnant to be center-filled in narrowband imaging of highionization lines (such as [O III] at $5007 \AA$ or He II at $4686 \AA$ ) and limb-brightened for low-ionization lines (such as [S II] at $6717 \AA$ ). The nonrelativistic blast wave does not reach the outer edge of young remnants and might be visible in a deep exposure of a high-resolution image. There might also be synchrotron emission in the radio band from the accelerated electrons in this shock. More interestingly, GRB remnants are expected to show ionization cones if the early UV afterglow emission from GRBs is beamed. The hydrodynamic spreading of the photoionized gas is negligible, as the gas expands at the sound speed of $\sim 10^{2} \mathrm{~km} \mathrm{~s}^{-1}$ and can traverse only a distance of $\lesssim 5 \mathrm{pc}\left(t_{L} / 5 \times 10^{4} \mathrm{yr}\right)$ during the lifetime of the remnant. This distance is at least an order of 
magnitude smaller than the remnant radius, so lateral expansion could smooth only extreme beaming factors of $f_{b} \lesssim\left(0.1^{2} / 4 \pi\right)=10^{-3}$.

\section{CONCLUSIONS}

We have computed the emission spectrum that results from the cooling and recombination of an interstellar medium whose equilibrium state has been altered by a GRB and its subsequent afterglow emission. We have identified some generic signatures that are quite likely to bear the footprints of a GRB, and whose close study in nearby galaxies can, in turn, give us direct information on the sites where GRBs typically occur and, maybe, lead us to the discovery of the remnant (if there is one) of the object which triggered the initial burst.

The X-ray emission is very weak compared to the UV and optical. This property could help separate GRBs from sources that provide a more steady energy supply, such as multiple supernovae or stellar winds; the latter type of sources tend to fill their remnants with hot X-ray-emitting gas.

We have found that the [O $\mathrm{III}] \lambda 5007$ to $\mathrm{H} \beta$ line ratio is indicative of the high values of the ionization parameter in GRB remnants (see Figs. 4 and 5). Detection of this and similar generic lines (see Fig. 3) at an $\mathrm{S} / \mathrm{N}=100$ is feasible for remnants in the Virgo Cluster after an hour of integration time with the Keck $10 \mathrm{~m}$ telescope. Narrowband imaging of such remnants could resolve the shock from the photoionized region inside these remnants and should reveal ionization cones if the early (prompt or afterglow) $\mathrm{UV}$ emission from GRBs is beamed.

We thank J. Huchra for useful discussions. A. L. and R. P. were supported in part by NASA grants NAG 5-7039 and NAG 5-7768. J. R. was supported in part by NASA grant NAG 5-2845.
Abbott, D. C., Bieging, J. H., \& Churchwell, E. 1981, ApJ, 250, 645 Akerlof, C. W., et al. 1999, Nature, 398, 400

Anders, E., \& Grevesse, N. 1989, Geochim. Cosmochim. Acta, 53, 197

Baldwin, J. A., Phillips, M. M., \& Terlevich, R. 1981, PASP, 93, 5

Baum, S. A., Heckman, T. M., \& van Breugel, W. 1992, ApJ, 389, 208

Bloom, J. S., et al. 1999, ApJ, 518, L1

Böttcher, M., Dermer, C. D., Crider, A. W., \& Liang, E. P. 1999, A\&A, 343, 111

Burgess, A. 1965, ApJ, 141, 1588

Cox, D. P. 1972, ApJ, 178, 143

Cox, D. P., \& Raymond, J. C. 1985, ApJ, 298, 651

Dopita, M. A., \& Sutherland, R. S. 1995, ApJ, 455, 468

Eichler, D., Livio, M., Pizan, T., \& Sehzamm, D. N. 1989, Nature, 340, 126

Efremov, Y. N., Elmegreen, B. G., \& Hodge, P. W. 1998, ApJ, 501, L163

Elmegreen, B. G., \& Chiang, W.-H. 1982, ApJ, 253, 666

Fenimore, E. E., \& Bloom, J. S. 1995, ApJ, 453, 25

Fruchter, A. S., et al. 1998, to appear in Proc. of the 4th Huntsville Symp. on Gamma-Ray Bursts, ed. C. A. Meegan, R. Preece, \& T. Koshut, (New York: AIP), 509

Galama, T. J., et al. 1998, ApJ, 500, L101

Ghisellini, G., Haardt, F. Campana, S. Lazzati, D., \& Covino, S. 1998, ApJ, 517,168

Hartigan, P. Raymond, J., \& Hartmann, L. 1987, ApJ, 316, 323

Jacobs, V. L., Davis, J., Kepple, P. C., \& Blaha, M. 1977, ApJ, 211, 605

Loeb, A., \& Perna, R. 1998, ApJ, 503, L35

MacFadyen, A., \& Woosley, S. E. 1999, ApJ, 524, 262

McCray, R., \& Kafatos, M. 1987, ApJ, 317, 190

Meszaros, P., \& Rees, M. J. 1997, ApJ, 476, 232

. 1998, MNRAS, 299, L10

\section{REFERENCES}

Morse, J. A. et al. 1996, AJ, 112, 509

Narayan, R., Paczyński, B., \& Pizan, T. 1992, ApJ, 395, 183

Paczyński, B. 1998, ApJ, 494, L45

Paczyński, B., \& Rhoads, J. E. 1993, ApJ, 418, L5

Perna, R., \& Loeb, A. 1998, ApJ, 501, 467

Raymond, J. C. 1979, ApJS, 39, 1

Raymond, J. C. et al. 1988, ApJ, 324, 869

Reilman, R. F., \& Manson, S. T. 1979, ApJS, 40, 815

Rhode, K. L., Salzer, J. J., Westfahl, D. J., \& Radice, L. 1999, AJ, in press, (astro-ph/9904065)

Ruffert, M., \& Janka, H.-T. 1999, A\&A, 366, 573

Sari, R. 1997, ApJ, 489, L37

Sedov, L. I. 1959, Dimensional Methods in Mechanics (New York: Academic)

Shull, J. M., \& McKee, C. F. 1979, ApJ, 227, 131

Smith, B. W., Mann, J. B., Cowan, R. D., \& Raymond, J. 1985, ApJ, 298, 898

Tenorio-Tagle, G. 1981, A\&A, 94, 338

Vietri, M. 1997a, ApJ, 478, L9

. 1997b, ApJ, 488, L105

Younger, S. E. 1981, J. Quant. Spectrisc, Radiat. Transfer, 26, 329

Wang, Q. D. 1999, ApJ, 517, L27

Waxman, E. 1997a, ApJ, 485, L5

. 1997b, ApJ, 489, L33

Wijers, R. A. M. J., Bloom, J. S., Bagla, J. S., \& Natarajan, P. 1998, MNRAS, 294, L13

Wijers, R. A. M. J., \& Galama, T. J. 1999, ApJ, 523, 1

Wijers, R. A. M. J., Rees, M. J., \& Meszaros, P. 1997, MNRAS, 288, L51

Woosley, S. E. 1993, ApJ, 405, 273 